\title{
Um modelo RCPSP para Gestão Ágil Scrum
}

\author{
Osmar Leandro Dantas da Silva ${ }^{1}$, Emanuel Dantas Filho ${ }^{2}$, Diego Rocha Lima ${ }^{1}$ \\ ${ }^{1}$ Instituto Federal do Ceará campus Crato (IFCE) \\ Caixa Postal 63115-500 - Crato - CE - Brasil \\ ${ }^{2}$ Instituto Federal da Paraíba campus Monteiro (IFPB) \\ Caixa Postal 58500-000 - Monteiro - PB - Brasil \\ osmarleandrods@gmail.com, emanuel.filho@ifpb.edu.br, \\ diego.rochadifce.edu.br
}

\begin{abstract}
The RCPSP is part of combinatorial problems of class scheduling, which seeks to optimize limited human resources in a project. The purpose of this research is to evaluate the adoption of a mathematical model for the RCPSP in agile management of a team of software development. The analyzed scenario consists to use the model in the allocation of teams using the Scrum methodology. As result, simulations were realized on the distribution activities of Sprints resources, and the proposed model indicated allocations with a gain of up to $16.60 \%$ in the project duration.
\end{abstract}

Resumo. O RCPSP faz parte da classe de problemas combinatórios de escalonamento, onde busca-se otimizar os recursos humanos limitados em um projeto. O propósito dessa pesquisa é avaliar a adoção de um modelo matemático para o RCPSP na gestão ágil de uma equipe de desenvolvimento de software. O cenário analisado consiste em utilizar o modelo na alocação de equipes que utilizam a metodologia Scrum. Como resultados, foram realizados simulações na distribuição de atividades dos recursos em Sprints, e o modelo proposto indicou alocações com ganho de até 16,60\% no tempo de duração de projetos.

\section{Introdução}

Para Versionone (2008), o uso de metodologias ágeis tem sido crescente em equipes de desenvolvimento de software, apresentando resultados positivos em termos de prazo, custo e qualidade. Tais abordagens visam à desburocratização das atividades (SCHWABER, 2013) e empregam princípios como ciclos iterativos e entregas frequentes de software (BECK, 2001).

No universo da Pesquisa Operacional (PO), o problema de alocação de recursos humanos em projetos (RCPSP) é conhecido como uma variação do problema do escalonamento. Para resolver problemas que envolvem PO, pode-se utilizar algoritmos exatos ou heurísticos.

O objetivo deste trabalho consiste em avaliar o uso de um modelo matemático para realizar a otimização de alocações na gestão de um Scrum Team. Para avaliar os resultados, foi comparado as alocações simuladas pelo modelo com alocações reais em equipes de projetos de uma fábrica de software. 


\section{Trabalhos Relacionados}

Em Kolisch (2006), o problema RCPSP é tratado para a minimização de dias do projeto (makespan), e maximização do valor presente líquido (VPL). Em Rodrigues (2010) temos uma pesquisa para otimização de tempo e custos na alocação de recursos em projetos usando o algoritmo MBA (Minimum Bounding Algorithm).

$\mathrm{Na}$ literatura, existem estudos de diversas meta-heurísticas e heurísticas que são usadas para resolver o problema de alocação de recursos em projetos. Pode-se citar as alocações baseadas na Busca Tabu, Simulated Annealing e Algoritmos Genéticos, respectivamente dos trabalhos de Baar (1999), Bouleimen (2003) e Ranjbar (2008).

Em projetos de desenvolvimento de software, existem restrições específicas que precisam ser tratadas no problema RCPSP. Algumas dessas restrições, como a alocação de recursos fracionados e/ou recursos heterogêneos, são mencionadas no trabalho de Filho e Gomes (2014).

\section{Metodologia}

Inicialmente foi realizado um estudo sistemático sobre Pesquisa Operacional, Programação Linear Inteira, e trabalhos relacionados do problema RCPSP. Em seguida foi adaptado o modelo matemático proposto por Filho e Gomes (2014) com adição de restrições específicas para alocações de equipes de desenvolvimento Scrum. Por fim, para subsidiar os resultados desta pesquisa, foram instanciados cenários utilizando o modelo e comparado com as alocações reais de uma equipe.

Com relação aos objetivos e procedimentos, a pesquisa é explicativa e experimental. $\mathrm{O}$ objeto de estudo corresponde à otimização de tempo na alocação de recursos humanos em equipes de desenvolvimento que utilizam a metodologia Scrum. Para avaliar os resultado do modelo matemático proposto, foram instanciados no software LINGO algumas Sprints presentes no backlog de uma fábrica de software.

\section{Resultados}

O modelo RCPSP desta pesquisa é uma adaptação ao modelo proposto por Filho e Gomes (2014) para satisfazer o cenário de equipes Scrum. Foram adicionados ao modelo as restrições dispostas nas equações 4.1 e 4.2 .

$$
\begin{array}{r}
F(a)=\left(\sum_{r, a \in R_{A}} M_{r a}+\sum_{a, b \in c_{\text {critico }}} S_{a b}\right)-1, \quad \forall a \in A \\
S(a, p)=\left(\sum_{p \in c_{\text {normal }}} F_{p}\right)+1, \quad \forall(a, p) \in C_{\text {Critico }}
\end{array}
$$

$\mathrm{Na}$ equação 4.1, para cada atividade (a), seu instante de término (F) deverá ser a soma de seus instantes de execução (M) com o momento de seu início (S), subtraindo-se o instante atual. Essa restrição é necessária para que o modelo refaça o caminho crítico das atividades do projeto.

Seguindo essa abordagem, a equação 4.2 mostra que para cada atividade (a), seu instante de início (S) é o próximo instante de tempo após o término (F) da sua atividade precedente (p). Portanto, deve-se definir previamente o início das atividades e suas precedências, que são representados pelo conjunto $S(a, p)$. 
Para avaliar os resultados do modelo, foi realizada uma análise comparativa da alocação real de uma equipe Scrum e a alocação proposta na simulação utilizando o modelo matemático RCPSP. Para tanto, foi selecionado um projeto em execução de uma fábrica de software que utiliza a metodologia Scrum. Esse projeto serviu de subsídios na avaliação dos resultados da pesquisa.

As estimativas das atividades e a escolha dos recursos são tarefas realizadas de forma manual pela equipe e conduzida pelo Scrum Master, caracterizando uma alocação real. Este mesmo cenário de recursos e atividades foi instanciado no modelo matemático. Para as simulações de alocações foi utilizado a versão de testes do solver Lingo 15.0, gentilmente cedida pelo LINDO Systems para a pesquisa.

A primeira instância analisada corresponde a uma Sprint 01 com duração estimada de 120 horas ( 3 semanas). Nessa Sprint foram alocadas trinta e três atividades para os seis recursos humanos disponíveis no projeto. A alocação real foi proposta pelo Scrum Team durante uma Sprint Planning. O mesmo backlog foi instanciado no modelo RCPSP e os recursos humanos cadastrados.

Utilizando o solver Lingo, o modelo gerou uma alocação para as mesmas atividades e propôs uma alocação com apenas 103 horas de duração. Importante frisar que todas as 33 atividades foram contempladas na alocação simulada pelo modelo.

Seguindo a mesma abordagem foram instanciadas as próximas seis Sprints do projeto. O gráfico com os resultados da análise comparativa, entre a alocação real e o a alocação simulada pode ser observada na Figura 1.

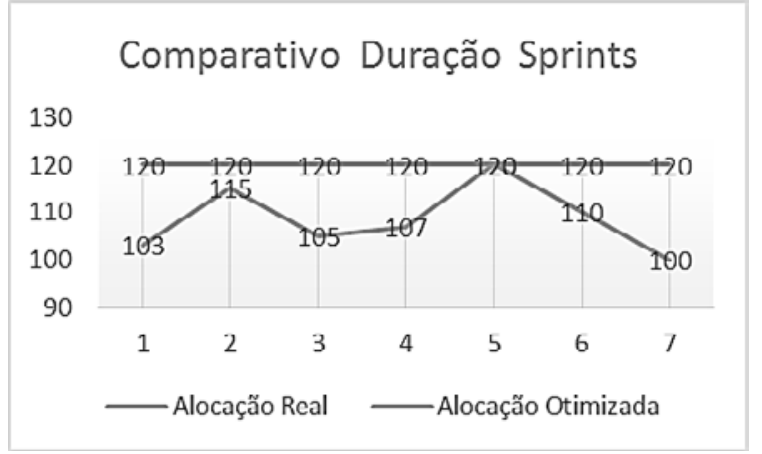

Figure 1. Comparativo de duração das Sprints.

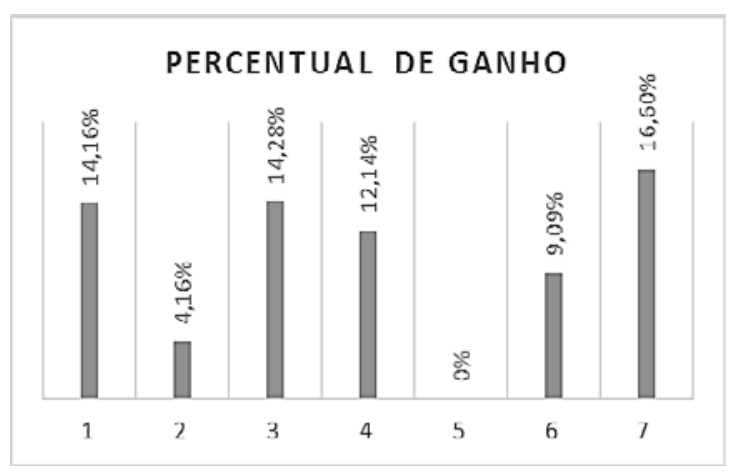

Figure 2. Percentual de ganho com o modelo.

Como visto no gráfico da Figura 1, em todas as Sprints analisadas o tempo de duração proposto pelo modelo RCPSP foi menor ou equivalente ao tempo de duração na alocação real. A Sprint 05 teve o tempo de planejamento igual ao tempo proposto na simulação do modelo matemático. Já na Sprint 07 foi obtido o melhor resultado. Nesta, o modelo propôs uma alocação com 100 horas, a maior discrepância com a alocação real dos cenários analisados.

Na Figura 2 temos a ilustração dos percentuais de ganho para cada Sprint. Esses resultados mostram a vulnerabilidade de executar alocações de forma manual, que é o processo utilizado atualmente pela equipe em questão. $O$ ganho em cada Sprint é uma relação percentual da quantidade de horas do planejamento real, com a quantidade de horas proposta pela simulação do mesmo cenário com o modelo RCPSP.

Ao otimizar o uso dos recursos, além de eliminar situações de atividades paralelas, o modelo proposto busca balancear a carga horária dos recursos. Outra situação tratada consiste em alocar para cada atividade o recurso com mais afinidade disponível. 


\section{Conclusão}

Como observado nos resultados, pode-se concluir que um modelo para automatizar e otimizar a alocação de equipes na gestão de projetos ágeis é de suma importância para área de TI. Vale observar que o problema investigado trata-se de um problema NP-hard, e que a alocação realizada de forma manual normalmente apresenta altas taxas de erros.

O modelo RCPSP busca otimizar o uso dos recursos, normalmente alocações realizadas sem auxílio de uma ferramenta tornam o processo susceptíveis a falhas. Visto a grande quantidade de variáveis a se analisar, realizar alocações é uma tarefa custosa e passível de erros constantes na gestão de projetos que utilizam a metodologia ágil Scrum.

Os resultados obtidos nesta pesquisa foram satisfatórios e cumpriram as expectativas de apresentar alocações com diminuição de tempo na distribuição das atividades para um Scrum Team. Propõe-se como trabalho futuro avaliar a aderência do modelo em outras equipes de desenvolvimento de software que utilizem a metodologia Scrum.

\section{Agradecimentos}

Nossos agradecimentos ao Instituto Federal do Ceará pelo financiamento da pesquisa e para LINDO Systems que disponibilizou a licença do solver Lingo para essa pesquisa.

\section{Referências}

Baar, T., Brucker, P., \& Knust, S. (1999) "Tabu search algorithms and lower bounds for the resource-constrained project scheduling problem", Springer US, pages 1-18.

Beck, K at all. (2001) "Manifesto for Agile Software Development", http://www.agilemanifesto.org, Fevereiro 2015.

Bouleimen, K., Lecocq, H. (2003) "A new efficient simulated annealing algorithm for the resource - constrained project scheduling problem and its multiple modes version", European Journal of Operational Research, 149(2), 268-281.

Filho, E. D., and Gomes, M. J. N. (2015) Modelos para Alocação de Recursos Humanos de Diferentes Perfis em Projetos de TI, In Gestão e Projetos: GeP, 6(1), 63-78.

Kolisch, R., and Hartmann, S. (2006) "Experimental investigation of heuristics for resource-constrained project scheduling: An update", European journal of operational research, 174(1), 23-37.

Ranjbar, M., Kianfar, F., and Shadrokh, S. (2008) "Solving the resource availability cost problem in project scheduling by path relinking and genetic algorithm". Applied Mathematics and Computation, 196(2), 879-888.

Rodrigues, S. B., and Yamashita, D. S. (2010) An exact algorithm for minimizing resource availability costs in project scheduling. In European Journal of Operational Research, 206(3), 562-568.

Schwaber, K.; Sutherland, J. (2013) "Guia do Scrum: Um guia definitivo para o Scrum: As regras do jogo". [Sl].

Versionone. (2008) The State of Agile Development Survey Results. http://www.versionone.com/pdf/3rdAnnualStateOfAgile_FullDataReport.pdf, Janeiro 2015. 